\title{
Correction to: Carbon-Based Metal-Free Electrocatalysis for Energy Conversion, Energy Storage, and Environmental Protection
}

\author{
Chuangang $\mathrm{Hu}^{1} \cdot$ Ying Xiao ${ }^{1,2} \cdot$ Yuqin $\mathrm{Zou}^{2} \cdot$ Liming Dai ${ }^{1,2,3}$
}

Published online: 5 April 2018

๑) Shanghai University and Periodicals Agency of Shanghai University 2018

\section{Correction to: Electrochemical Energy Reviews https://doi.org/10.1007/s41918-018-0003-2}

The original version of this article unfortunately contained mistakes. The title was incorrect. The corrected title is given above.

The original article has been corrected.

The original article can be found online at https://doi.org/10.1007/ s41918-018-0003-2.

Liming Dai

liming.dai@case.edu

1 Center of Advanced Science and Engineering for Carbon (Case4Carbon), Department of Macromolecular Science and Engineering, Case Western Reserve University (CWRU), Cleveland, OH 44106, USA

2 BUCT-CWRU International Joint Laboratories, College of Energy, Beijing University of Chemical Technology (BUCT), Beijing 100029, China

3 UNSW-CWRU International Joint Laboratories, School of Chemical Engineering, University of New South Wales (UNSW), Sydney, NSW 2025, Australia 